\title{
Formation of high-order acoustic Bessel beams by spiral diffraction gratings
}

\author{
Noé Jiménez,,$^{1, *}$ R. Picó, ${ }^{2}$ V. Sánchez-Morcillo, ${ }^{2}$ V. Romero-García, ${ }^{1}$ L. M. García-Raffi, ${ }^{3}$ and K. Staliunas ${ }^{4}$ \\ ${ }^{1}$ LUNAM Université, Université du Maine, CNRS, LAUM UMR 6613, Avenue O. Messiaen, 72085 Le Mans, France \\ ${ }^{2}$ IGIC, Universitat Politècnica de València, Paranfimf, E-46730 Grao de Gandia, Spain \\ ${ }^{3}$ IUMPA, Universitat Politècnica de València, Camino de Vera s/n, 46022 València, Spain \\ ${ }^{4}$ ICREA, Departament de Física i Enginyeria Nuclear, Universitat Politècnica de Catalunya, Colom 11, E-08222 Terrasa, Barcelona, Spain
}

(Received 28 April 2016; published 23 November 2016)

\begin{abstract}
The formation of high-order Bessel beams by a passive acoustic device consisting of an Archimedes' spiral diffraction grating is theoretically, numerically, and experimentally reported in this paper. These beams are propagation-invariant solutions of the Helmholtz equation and are characterized by an azimuthal variation of the phase along its annular spectrum producing an acoustic vortex in the near field. In our system, the scattering of plane acoustic waves by the spiral grating leads to the formation of the acoustic vortex with zero pressure on axis and the angular phase dislocations characterized by the spiral geometry. The order of the generated Bessel beam and, as a consequence, the size of the generated vortex can be fixed by the number of arms in the spiral diffraction grating. The obtained results allow for obtaining Bessel beams with controllable vorticity by a passive device, which has potential applications in low-cost acoustic tweezers and acoustic radiation force devices.
\end{abstract}

DOI: 10.1103/PhysRevE.94.053004

\section{INTRODUCTION}

Diffraction of waves, the spreading of the wave packet upon propagation, represents one of the most known properties of wave physics. However, there exist particular solutions of the wave equation that are immune to diffraction. Among them, the Bessel beams [1] are diffraction-free solutions with remarkable features. Of special interest are the high-order Bessel beams (HOBBs), characterized by high amplitude concentric rings with a profile given by the $n$ th-order Bessel function on the plane transverse to the beam axis. Any Bessel beam is characterized by an annular radiation in the far field, therefore the magnitude of the spatial spectrum of these beams does not depend qualitatively on the order of the beam. The difference between zeroth order and HOBBs is that the phase of the HOBBs shows a linear variation along its annular spectrum in the azimuthal direction. Thus, the wave field presents a helicoid phase dependence containing screw-type phase singularities, leading to an intensity minimum at the beam axis. Solutions of such kinds are of infinite transverse extent and thus cannot be generated experimentally. However, it is possible to generate finite size approximations for Bessel beams which propagate over extended distances in a diffraction-free manner providing potential applications for the wave physics community [2-7]. Whereas zeroth-order Bessel beams present a bright central maximum and can be useful for applications that require focusing of energy [8], the vortex beams generated by the HOBBs can be useful for manipulating particles in both optics [9] and acoustics [10].

In the case of electromagnetic (optical) waves, vortex beams have experimentally been demonstrated by means of computer generated holograms $[2,11]$ or by axicons illuminated with a Laguerre-Gaussian mode [3]. Other methods include an azimuth-dependent retardation on the optical field using spiral phase plates (SPPs) [4] or diffraction gratings with groove

\footnotetext{
*noe.jimenez@univ-lemans.fr
}

bifurcation. In the latter case, vortex beams with an arbitrary topological charge have been created [12]. This kind of beam has been shown to be very useful for the optical manipulation of particles. Since the first observations of the manipulation of particles using optical beams [13-15], an unexpected radial force field appeared, called the gradient force, that dragged colloidal particles towards the axis in addition to the axial radiation pressure that pulls particles towards the beam. In this way, particles can be trapped into the beam axis under conditions where the dragging gradient force dominates over the pulling radiation pressure. This regime can be achieved using strongly focused light beams and for particles smaller than the wavelength leading to the possibility of manipulating objects as small as $5 \mathrm{~nm}$ [16]. These so-called optical tweezers have been employed in many other macromolecular, biological, and medical applications [16]. However, high intensity beams are necessary for exerting strong forces leading to unwanted effects as heating, so in practical applications optical tweezers can exert forces up to $100 \mathrm{pN}[16]$.

Compared to optical manipulation, ultrasonic waves become advantageous for manipulating heavier objects: The smallness of the sound speed leads to larger drag forces from 3 to 4 orders of magnitude [10], and because of the size of the acoustic wavelength, bigger objects can be trapped. In addition, the interaction of Bessel beams with particles has also been studied intensively in acoustics. Two main remarkable effects have been reported: On one hand, the transference of orbital momentum from the acoustical vortex to the particle $[17,18]$, and on the other hand, the appearance of negative axial acoustic radiation forces, observed first by Marston $[5,19]$. This fact has motivated the development of experimental approaches to generate acoustical vortex beams. Different methods using either single acoustic sources have been developed. Phase dislocations using a single source were first proposed by Nye and Berry [20]. The acoustic analog of the optical SPP has also been proposed [21,22], consisting of a transducer with a surface properly deformed to create the 
helicoidal beam. This method is restricted to a single operating frequency. Other approaches with single sources include the use of a photoacoustic effect to generate a helical beam [23] or the use of metasurfaces based on Helmholtz resonators to control the angular phase [24].

On the other hand, the generation of acoustical vortices with arrays of sources is also possible [25] and has widely been used in acoustics for multiple applications: particle manipulation [26], acoustical tweezers [10,27-29], angular momentum transfer [30], acoustic spanners [31], multipleparticle trapping [32], precise manipulation and sorting of cells for life sciences research $[33,34]$, or microbubble capturing [35]. Recently Baresch and co-workers developed the first all-acoustical single-beam trapping [10] where a negative gradient pulling force with acoustic waves was demonstrated using a single vortex beam. However, although the array of sources provides active steering and control of the vortex beam, in such active systems, the resolution of the vortex is restricted by the number of transducers in the array [36], leading to technologically complex systems in the case of vortices of high topological charge.

In this paper we study the diffraction of a plane wave by a multiple-arm Archimedes' spiral diffraction grating and propose a passive and robust method for the formation of HOBBs using such gratings. The scattering of plane acoustic waves by the spiral grating leads to the formation of the acoustic vortex with zero pressure on axis and the angular phase dislocations characterized by the spiral geometry. The order of the generated Bessel beam and, as a consequence, the size (width) of the generated vortex can be fixed by the number of arms of the spiral diffraction grating. The obtained results allow for obtaining Bessel beams with controllable vorticity by a passive device, which has potential applications in low-cost acoustic tweezers and acoustic radiation force devices. First, in Sec. II we present a theoretical model for the diffraction of plane waves by the multiple-arm spiral grating with infinite radial extent. Then we numerically analyze the effects of the finite size of the sample, considering also the effects of the vibration of the scatterers. The numerical confirmation of the HOBBs is reported in Sec. III showing the generation of the vortex in the axis and its dependence on the topological charge of the spiral diffraction grating. Finally, in Sec. IV we experimentally test the main results of this paper by measuring the acoustic field scattered by a steel grating embedded in water. Particularly, we show the phase dislocation and the acoustic vortex generation by a first-order Bessel beam.

\section{DIFFRACTION BY A SPIRAL GRATING}

The proposed structure is a multiple-arm Archimedes' spiral diffraction grating as shown in Figs. 1 and 2. As the arms of the Archimedes' spiral present a uniform separation, the incident field is diffracted at an angle, given by diffraction grating theory. Therefore, the diffracted field is of a conical wave front as in Ref. [7] but here with the azimuthal rotating phase due to the spiral geometry. When converging to the axis, the conical wave front forms a HOBB. The diffracted pressure field by the grating generates an acoustic vortex line with a characteristic screw dislocation.

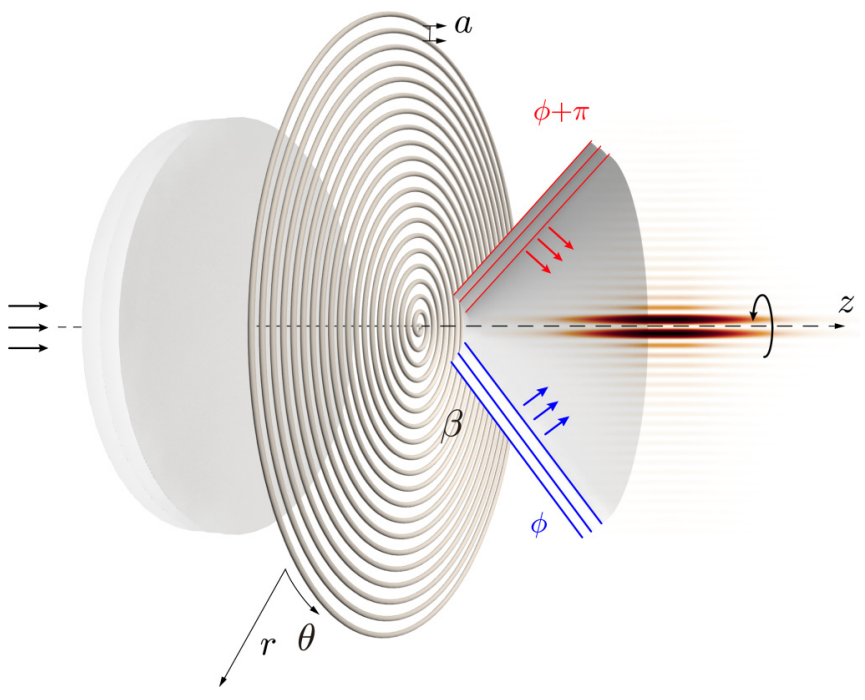

FIG. 1. Scheme of the first-order Bessel beam formation by the Archimedes' spiral grating. The incident plane wave is scattered into a converging and diverging conical wave front in which the phase of the wave $(\phi)$ is a linear function of the angle $(\theta)$. A corkscrew dislocation in the wave front is produced, leading to an acoustic vortex and a zero pressure on-axis $r=0$. The minimum along the symmetry axis results from the destructive interference of first-order diffraction generated at opposite sides of the axis.

\section{A. Infinite diffraction grating}

The harmonic pressure field diffracted by a grating with source velocity distribution $v_{z}\left(\mathbf{r}_{0}\right)$ can be calculated using the Rayleigh-Sommerfeld integral at any point in cylindrical coordinates $\mathbf{r}=(r, \theta, z)$ as

$$
p(\mathbf{r})=\frac{-i \omega \rho_{0}}{2 \pi} \int_{S_{0}} v_{z}\left(\mathbf{r}_{0}\right) \frac{\exp \left(i k\left|\mathbf{r}-\mathbf{r}_{0}\right|\right)}{\left|\mathbf{r}-\mathbf{r}_{0}\right|} d S\left(\mathbf{r}_{0}\right)
$$

where $\mathbf{r}_{0}=\left(r_{0}, \theta_{0}, z_{0}\right)$ is the radius vector of a surface element $d S, \omega$ is the angular frequency, the wave number is $k=\omega / c_{0}$, and $\rho_{0}$ and $c_{0}$ are the density and speed of sound of the medium. A time dependence $\exp (-i \omega t)$ was assumed. The plane of the source is assumed to be at the origin of the coordinates for simplicity $z_{0}=0$, then, without loss of generality we can write $v_{z}\left(r_{0}, \theta_{0}, z_{0}=0\right)=v_{z}\left(r_{0}, \theta_{0}\right)$.

The Fresnel approximations assume that the first two terms of the square root Taylor expansion are sufficient to correctly represent the phase, provided that $z$ is large enough (parabolic expansion),

$$
\begin{aligned}
\left|\mathbf{r}-\mathbf{r}_{0}\right| & =z \sqrt{1+\frac{\left(x-x_{0}\right)^{2}}{z^{2}}+\frac{\left(y-y_{0}\right)^{2}}{z^{2}}} \\
& \simeq z\left(1+\frac{r^{2}}{2 z^{2}}+\frac{r_{0}^{2}}{2 z^{2}}-\frac{r r_{0} \cos \left(\theta_{0}-\theta\right)}{z^{2}}\right)
\end{aligned}
$$

and by neglecting the radial contributions in the denominator of the Rayleigh integral: $\left|\mathbf{r}-\mathbf{r}_{0}\right| \simeq z$. 



FIG. 2. Examples of Archimedes' spirals with multiple arms (a) $n=1$, (b) $n=3$, (c) $n=7$, and (d) $n=20$.

Expressing the surface element in cylindrical coordinates $d S=r_{0} d \theta_{0} d r_{0}$, Eq. (1) transforms to the following form:

$$
\begin{aligned}
p(r, \theta, z)= & A(r, z) \int_{0}^{\infty} \int_{0}^{2 \pi} v_{z}\left(r_{0}, \theta_{0}\right) \exp \left[i \frac{k}{2 z} r_{0}^{2}\right] \\
& \times \exp \left[-i \frac{k r}{z} r_{0} \cos \left(\theta_{0}-\theta\right)\right] r_{0} d r_{0} d \theta_{0},
\end{aligned}
$$

where $A(r, z)$ is an independent expression on the integration variables $r_{0}$ and $\theta_{0}$,

$$
A(r, z)=\frac{-i \omega \rho_{0}}{2 \pi z} \exp \left[i k\left(z+\frac{r^{2}}{2 z}\right)\right] .
$$

We suppose that an incoming plane wave uniformly illuminates the spiral grating at $z=0$. The source velocity distribution can be characterized by a complex-amplitude transmission function [2],

$$
v_{z}\left(r_{0}, \theta_{0}\right)=v_{0} \exp \left[-i k_{r} r_{0}\right] \exp \left[-i \phi\left(\theta_{0}\right)\right],
$$

where $v_{0}$ is the particle velocity amplitude. The factor $\exp \left[-i k_{r} r_{0}\right]$ is the phase of the conical wave front. The continuity of the transversal component of the wave vector $k_{r}$ at the interface between the homogeneous medium and the diffraction grating with scatterers separated by a distance $a$ results in $k^{2}=\sqrt{k_{r}^{2}+k_{z}^{2}}$ with $k_{r}=2 \pi N / a$ and $k_{z}=k_{r} / \tan \beta$ as the axial and longitudinal wave numbers, respectively, $N$ is the diffraction order, and $\beta=\arcsin N \lambda / a$ is the angle of the conical wave front with respect to axis $z$. Here and below we will work in the range of frequencies that only excites the first diffraction order, therefore we assume $N=1$. The last factor $\exp [-i \phi(\theta)]$ is a phase accounting for the azimuthal dependence of the phase of the conical wave front.

In the case of a pure axisymmetric grating [7] where the sources are distributed in concentric circles separated at a

distance $a$,

$$
\begin{aligned}
\phi\left(\theta_{0}\right) & =k_{r} R\left(\theta_{0}\right)=\frac{2 \pi}{a} R\left(\theta_{0}\right)=\text { const. } \\
\exp \left[-i \phi\left(\theta_{0}\right)\right] & =\text { cte. }
\end{aligned}
$$

Therefore, due to the constant radius of each of the concentric rings, the phase is independent of azimuthal angles, and no vortex can be produced. In this paper we consider an Archimedes' spiral grating which provides the azimuthal dependence of the phase in our system. The general mathematical expression for a curve describing $n$ arms of Archimedes' spirals starting from an origin can be expressed in polar coordinates as

$$
R\left(\theta_{0}\right)=\frac{n a}{2 \pi} \theta_{0}+l a,
$$

with $0 \leqslant l \leqslant n-1$ as the index of the $l$ th arm and $a$ as the raidal separation between arms. Figure 2 shows examples of spirals with multiple arms. The phase term of Eq. (5) can be expressed as

$$
\exp \left[-i \phi\left(\theta_{0}\right)\right]=\exp \left[-i k_{r} R\left(\theta_{0}\right)\right]=\exp \left[-i n \theta_{0}\right] .
$$

The velocity function can be obtained by substituting Eq. (8) into Eq. (5),

$$
v_{z}\left(r_{0}, \theta_{0}\right)=v_{0} \exp \left[-i k_{r} r_{0}\right] \exp \left[-i n \theta_{0}\right] .
$$

Therefore, the particle velocity field at the source plane corresponds to a conical wave front with an azimuthal phase rotation proportional to the number of arms of the spiral. Explicitly, the phase of the field scattered by a $N$-arm spiral rotates by $2 \pi N$, thus, forming the phase singularity of the $N$ th order. The analysis of the other velocity function to obtain vortex beams can be found in the literature [37].

The pressure field can be obtained by substituting the source field velocity in the double integral in Eq. (3),

$$
\begin{aligned}
p(r, \theta, z)= & A(r, z) \int_{0}^{\infty} r_{0} \exp \left[\left(\frac{k}{2 z} r_{0}^{2}\right)\right] \\
& \times \int_{0}^{2 \pi} v_{0} \exp \left[-i k_{r} r_{0}\right] \exp \left[-i n \theta_{0}\right] \\
& \times \exp \left[i\left(\frac{k r}{z} r_{0} \cos \left(\theta_{0}-\theta\right)\right)\right] d r_{0} d \theta_{0} .
\end{aligned}
$$

The terms without azimuthal dependence that can be factorized out of the azimuthal integral in Eq. (10) are as follows:

$$
\begin{aligned}
p(r, \theta, z)= & A(r, z) v_{0} \int_{0}^{\infty} r_{0} \exp \left[i\left(\frac{k}{2 z} r_{0}^{2}-k_{r} r_{0}\right)\right] \\
& \times \int_{0}^{2 \pi} \exp \left[-i n \theta_{0}\right] \\
& \times \exp \left[-i \frac{k r}{z} r_{0} \cos \left(\theta_{0}-\theta\right)\right] d r_{0} d \theta_{0} .
\end{aligned}
$$

Using the Jacobi-Anger expansion,

$$
J_{n}(\alpha)=\frac{i^{n}}{2 \pi} \int_{0}^{2 \pi} \exp [i n \beta] \exp [-i \alpha \cos (\beta)] d \beta,
$$

and simple algebra with a change in variable, the integration over the azimuthal angle $\theta_{0}$ in Eq. (11) can be solved and leads 
to

$$
p(r, \theta, z)=B(r, z) F(r, z),
$$

where

$$
B(r, z)=A(r, z) 2 \pi v_{0} \exp \left[i n\left(\theta-\frac{\pi}{2}\right)\right]
$$

and

$$
F(r, z)=\int_{0}^{\infty} r_{0} \exp \left[i\left(\frac{k}{2 z} r_{0}^{2}-k_{r} r_{0}\right)\right] J_{n}\left(\frac{k r}{z} r_{0}\right) d r_{0}
$$

Note that $n$ is the number of arms in the spiral that is also the order of the Bessel function.

The radial integral in Eq. (15) can approximately be solved by using the method of the stationary phase $[2,3,38]$. Rapid oscillations of the exponential term of the integral in Eq. (15) mean that $F(r, z) \simeq 0$ over those regions and only significant nonzero contributions to the integral occur in regions of the integration range where the phase term is constant, i.e., at points of the stationary phase. In our case, the approximated solution to leading order of the radial integral at point $(r, \theta, z)$ reads as

$$
F(r, z) \simeq \frac{k_{r} z}{k} \exp \left[-i\left(\frac{k r^{2}}{2 z}+\frac{z k_{r}^{2}}{2 k}\right)\right] \sqrt{\frac{2 \pi z}{k}} J_{n}\left(k_{r} r\right) .
$$

Higher-order terms not considered in this solution give corrections to off-axis areas [38].

By substituting Eq. (16) into Eqs. (14) and (12) as well as using Eq. (4), the pressure field is written as

$$
p(\mathbf{r}) \simeq-i p_{0} k_{r} \sqrt{\frac{2 \pi z}{k}} J_{n}\left(k_{r} r\right) \exp \left[i k_{z} z\right] \exp [i n(\theta-\pi / 2)],
$$

where $p_{0}=\rho_{0} c_{0} v_{0}$ and the paraxial approximation of the axial wave number $k_{z}=k\left(1-k_{r}^{2} / 2 k^{2}\right)$ was used. The radial distribution is given by the $n$ th-order Bessel function, whereas the amplitude is proportional to $\sqrt{z}$, which is in fact the expression for a $n$ th-order Bessel beam. As an example we evaluate the amplitude of the pressure field along the first lobe of the first-order Bessel beam $(n=1)$ generated by an infinite spiral with $a / \lambda=1.2$ embedded in water. The black dashed line in Fig. 3(a) shows the evaluation of Eq. (17) for this spiral, showing the $\sqrt{z}$ dependence. Notice that the normalized intensity $p p^{*} / \rho_{0} c_{0}$ grows linearly with distance $z$ with a rate given by $2 \pi k_{r}^{2} J_{n}^{2}\left(k_{r} r\right) / k$. A simple physical interpretation of the $\sqrt{(z)}$ dependence in Eq. (17) is that the radiation at increasing $z$ arrives scattered from the arms of spirals of the increasing radius with proportionally increasing energy.

\section{B. Finite size effects}

The previous section deals with an infinitely extended diffraction grating. This is not the real situation; in experiments finite size effects are present. In order to analyze these finite size effects we have applied two different methods. On one hand we have numerically integrated the RayleighSommerfeld diffraction integral Eq. (1) for structures with finite extent. This allows for studying the effects due to the finite radial size of the spiral. On the other hand, numerical simulations using a three-dimensional (3D) pseudospectral time-domain method using a $k$-space corrector operator [39]
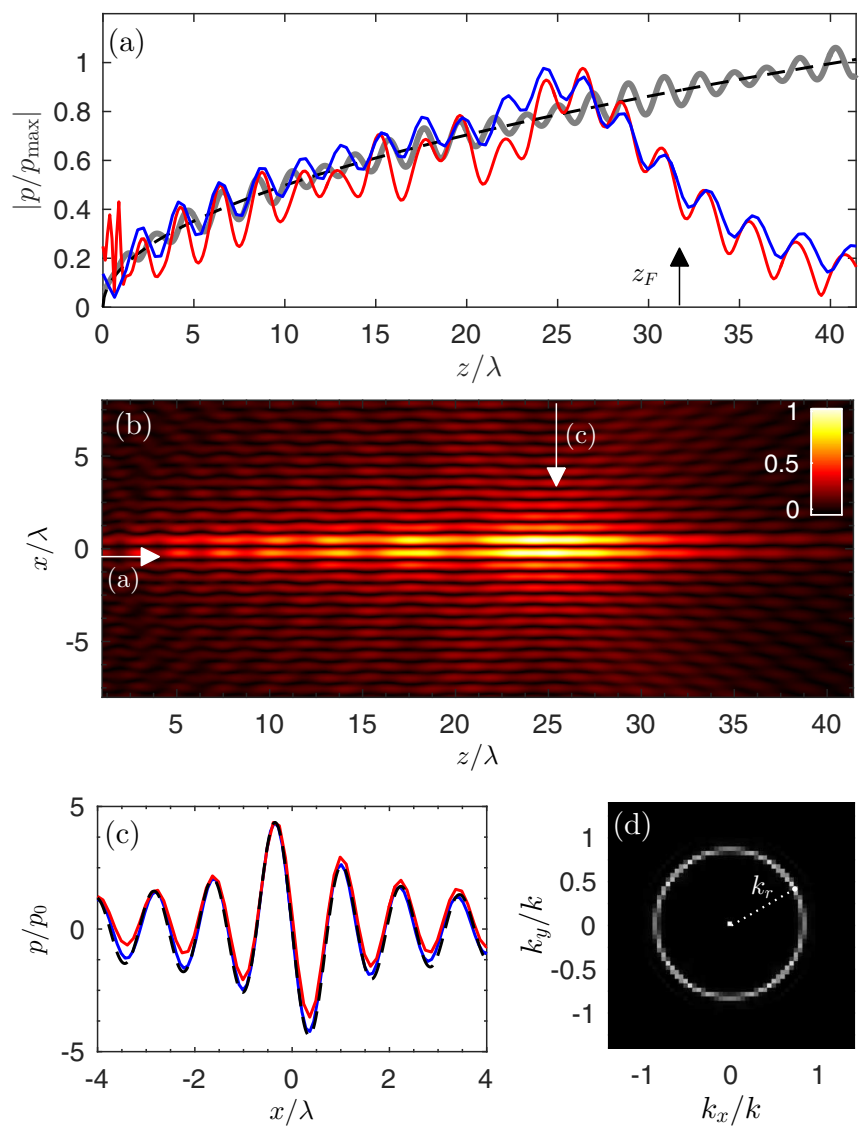

FIG. 3. (a) Longitudinal pressure distribution along the first lobe of the Bessel beam obtained from Eq. (1) for (blue) $M=$ 40 , (gray) $M=1000$, (red) $k$-space simulation considering elastic scatters, and (black dashed) analytic Eq. (17). (b) Pressure map distribution obtained by numerical evaluation of the RayleighSommerfeld diffraction integral Eq. (1) for a spiral of $M=40$. (c) Transversal pressure distribution at $z / \lambda=25$ obtained from Eq. (1) for (blue) $M=40$, (red) $k$-space simulation considering elastic scatters, and (black dashed) analytic Eq. (17). (d) Far field showing the characteristic ring of the Bessel beam, where $k_{r}=2 \pi / a$.

were also performed. In these simulations, a steel spiral grating embedded in water is considered, allowing the acoustic waves to penetrate in the grating's bulk material, so the physical compressibility and impedance of the material were considered.

Figure 3(b) shows the pressure field from the spiral grating analyzed in the previous section as obtained now by numerical integration of the Rayleigh-Sommerfeld diffraction integral Eq. (1) for a finite spiral structure of $M=40$ loops $(R=40 a)$. The formation of a first-order Bessel beam with the elongated zero field at the axis can be observed. An axial cross section along the first lobe of the Bessel beam is shown in Fig. 3(a) (blue line). This numerical integration of the Rayleigh-Sommerfeld integral agrees well with theory (black dashed line). It is worth noting here that the longitudinal field oscillations, observed also in Bessel beams generated by axicons, are not present in Eq. (17) as long this result was derived for a nontruncated spiral $M \rightarrow \infty$. As the number of spiral loops increases, the longitudinal oscillations tend 
to disappear, and the field converges to the one given by Eq. (17). To prove this we have evaluated a spiral grating with $M^{\prime}=1000$ with the same radius as the previous spiral (i.e., the distance between the scatterers, $a^{\prime}$ 's scaled, respectively, $R=M^{\prime} a^{\prime}=40 a$ ); the gray curve in Fig. 3(a) clearly shows this convergence.

Furthermore, the beam amplitude follows Eq. (17) increasingly along $z$ from $z=0$. However, as shown in Fig. 3(a) for the case of the truncated systems (blue line) the amplitude grows up to a given distance $z=z_{F}$. This distance can be estimated geometrically through the zeroth-order Bessel beams as [7]

$$
z_{F}=\frac{R a}{N \lambda} \sqrt{1-\left(\frac{N \lambda}{a}\right)^{2}},
$$

where $N \in \mathbb{N}$ is the diffraction order and $R=M a$ is the radius of the spiral with $M$ as the windings. The $z_{F}$ for the spiral with $M=40$ is shown in Fig. 3(a) whereas for the case with $M=1000$ it is out of the limits of the plotted frequencies. The estimation of $z_{F}$ is in good agreement with the numerical integration of Eq. (1).

In the radial direction, the beam follows a Bessel profile Eq. (17). A transversal cross section at $z / \lambda=25$ and $y=0$ is presented in Fig. 3(c), showing good agreement between the radial field distribution obtained by the numerical integration and theory. In order to prove this, Fig. 3(d) shows the far field of the truncated grating where the characteristic ring of a Bessel beam is observed, corresponding to the wave vector $k_{r}=2 \pi / a$.

Finally, we analyze the effect of the rigidity of the material of the spiral grating on the scattered field using numerical simulations. We analyze the scattering by the spiral grating with $M=40 a$ radiated by a plane wave. In these simulations, no losses were included, and the thickness of the grating was $\lambda / 4$. The simulation result, the red curve in Fig. 3(a), presents axial oscillations due to the finite aperture of the grating as already predicted by the direct integral of Eq. (1). Here, excellent agreement is found between simulations and theory. The small discrepancies are caused by the fact that the scatterers are of finite size, i.e., not the perfect punctual sources (as assumed in the Rayleigh-Sommerfeld integral) and due to the bulk resonances into the body of the steel grating. The radial profile, shown in Fig. 3(c), also shows the Bessel profile in good agreement with the theory and the direct integration of Eq. (1). Therefore, the effect of the rigidity of a steel grating embedded in water is small.

\section{HIGH-ORDER BESSEL BEAMS}

One of the consequences of the procedure described above is that it is possible to generate HOBBs by using multiple-arm spirals $(n>1)$. The parametric Eq. (7) describes $n$ arms, separated by a fixed distance $a$. In the case of $n>1$, the rate of growth of each individual arm is increased by a factor of $n$ as it is underlined by the dark arms in Fig. 2. The variation of the phase $(\phi)$ with the angle $(\theta)$ is therefore increased, and the conical wave front formed by the axisymmetric grating presents a total phase shift of $2 \pi n$ over a complete turn as follows from Eq. (9). When converging to the axis, the conical wave front forms a Bessel beam in the same way as in the previous section. However, as the phase rotation is increased, the vortex presents a topological charge of $n$. The conformed Bessel beam is therefore a $n$ th-order Bessel beam, and as a consequence the hollow central area of the beam is extended.

Figure 4 presents the formation of $\mathrm{HOBBs}$ for the cases of $n=3,7,20$ and 50. In Figs. 4(a)-4(d), the transversal pressure distribution at $z=z_{F}$ and $y=0$ is presented. In this case, the solid line presents the analytical solution for the transverse field of an ideal Bessel beam $J_{n}\left(k_{r} r\right)$, and the symbols are the $k$ space numerical solutions assuming a steel grating embedded in water. The simulations are in agreement with theory, even in the case of $n=50$. Notice here the discrepancies at high radial values in which the approximated theoretical solution fails because corrections of higher order should be taken into account, and the finite size effect of the sample is noticed in these regions.

The complete transversal map at $z=z_{F}$ is shown in Figs. 4(e)-4(h). When the areas of strongly reduced sound around the symmetry axis appear, the larger is $n$, the larger are the zero-field areas. The radius of the reduced sound areas can be estimated from the position of the first maximum of the $n$th Bessel function as

$$
r_{n}=\frac{j_{n}^{\prime} a}{2 \pi} \simeq \frac{\left(n+0.8086 n^{1 / 3}\right) a}{2 \pi},
$$

where $j_{n}^{\prime}$ is the first zero of the first derivative of the $n$th Bessel function [40]. For the case of $n=7, j_{n}^{\prime} \simeq 8.57$, therefore $r_{7} / \lambda=1.64$ in agreement with the results shown in Fig. 4(b). The axial map of the field shown in Figs. 4(i)-4(1) also shows the dependence of the hollow central part of the beam on the increasing order $n$. Of special interest is the generation of high-order beams, e.g., $n=50$. In this case, $j_{n}^{\prime} \simeq 57.12$, therefore the zero in the center of the hollow beam covers a cylindrical volume with a diameter of $2 r_{50} / \lambda=21.82$ in the interior of which the scattered sound is almost absent.

Finally, the phase of the field is presented in the subpanels Figs. 4(m)-4(p) at $z=z_{F}$. It can be seen that the number of times the phase rotates in each turn, i.e., the topological charge, is proportional to the order of the Bessel beam, in accordance with Eq. (17). The formed fields are therefore vortex beams of topological charge $n$ where the topological charge of the vortex can be controlled directly by the number of arms of the spiral grating.

These results show that HOBBs can be generated even by acoustically permeable gratings for a water-to-steel impedance contrast ratio, i.e., under realistic conditions for common ultrasound applications.

\section{EXPERIMENTAL VALIDATION: A FIRST-ORDER BESSEL BEAM FORMED BY A SPIRAL GRATING}

A spiral grating in water is studied experimentally in order to create an acoustic vortex by this kind of passive structure. A spiral profile in a stainless steel plate of $0.8 \mathrm{~mm}$ thickness was laser cut. The diameter of the scattering area was $\Delta r=0.75 \mathrm{~mm}$ and the grating period of $a=1 \mathrm{~mm}$. The width of the open slits is $a-\Delta r=0.25 \mathrm{~mm}$ for the open slits. The spiral winds $M=20$ times, and the total radius 

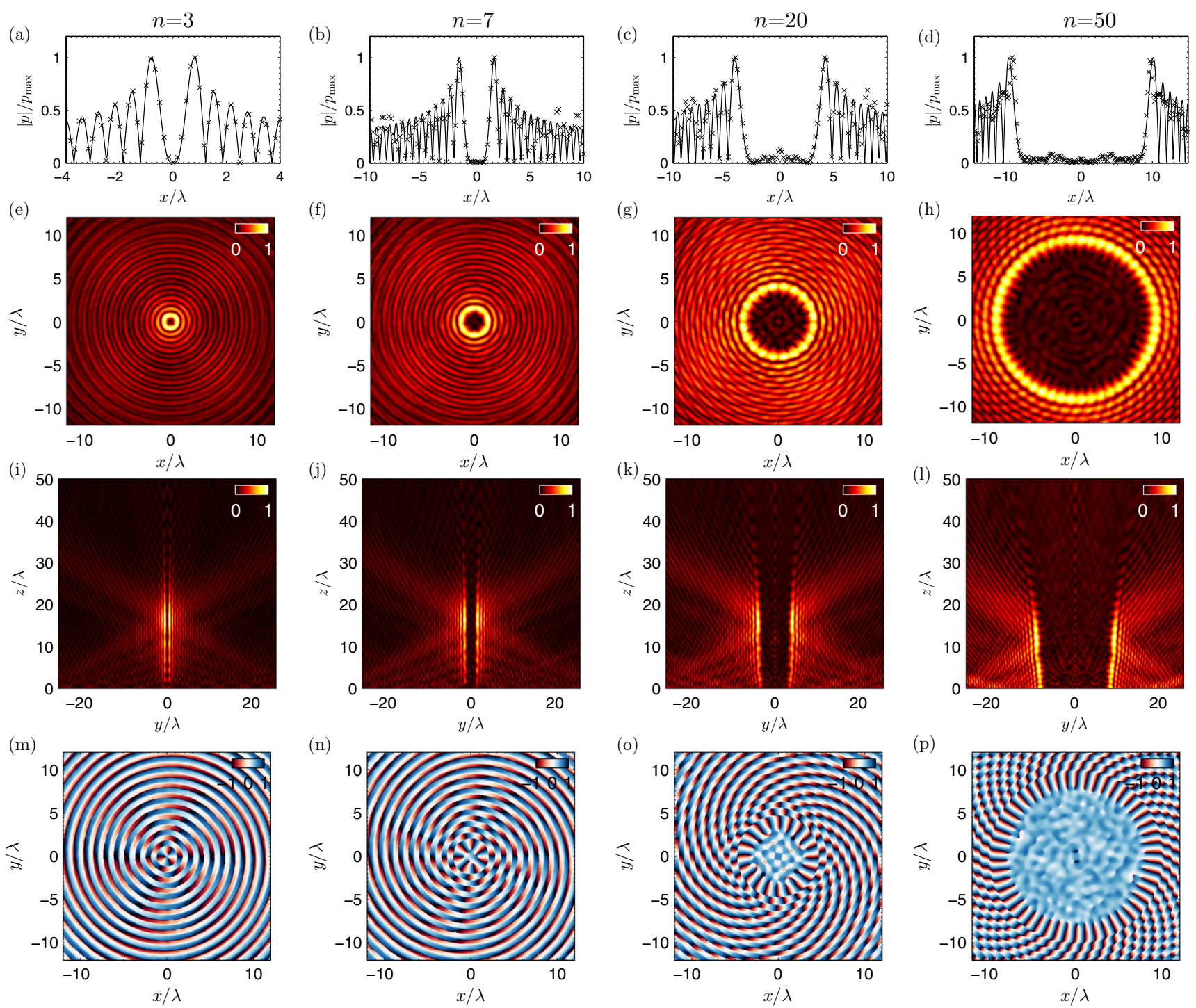

FIG. 4. $n$th order Bessel beams formed by spiral gratings of $n$ arms. (a)-(d) Transversal pressure distribution at $z=z_{F}$ and $y=0$, (continuous line) Eq. (17), and (markers) $k$-space numerical solution of the wave equation assuming a steel grating embedded in water. Pressure magnitude obtained at (e)-(h) $z=F$ and (i)-(l) $x=0$. Color bars in normalized units $p / p_{\max }$. (m) $-(\mathrm{p})$ The phase of the field is calculated at $z=z_{F} ;$ color bars are in normalized units of $\phi / \pi$.

is $R=50 \mathrm{~mm}$. The spiral plate is aligned and placed in front of a flat ultrasonic transducer of the same diameter as the grating as can be seen in Fig. 5(a). The source was driven by a 50 cycle sinusoidal pulse burst of a frequency of $f_{0}=2.22 \mathrm{MHz}$ using a function generator (14 bits, $100 \mathrm{MS} / \mathrm{s}$, model PXI5412, National Instruments) and a linear rf amplifier (ENI 1040 L, 400 W, 55 dB, ENI, Rochester, NY). The pressure wave forms were recorded with the help of a HNR $500 \mu \mathrm{m}$ needle polymer polyvinylidenfluorid hydrophone (Onda Corp., CA), and a digitizer (64 MS/s, model PXI5620, National Instruments) was used. A three-axis micropositioning system (OWIS GmbH, Germany) was used to move the hydrophone in three orthogonal directions with an accuracy of $10 \mu \mathrm{m}$, and a National Instruments PXI-Technology controller NI8176 was used to control all the devices. As Fig. 5(a) shows, the grating was placed in the near field just above the flat piezoelectric element at a distance of $0.5 \mathrm{~mm}$. Because of this gap, cavity resonances between the diffraction plate and the source appear. These resonances cause beam aberrations, however, as we will show below, the main features of the HOBB are retained.

The pressure field is represented in Fig. 5(b) by the numerical integration of Eq. (1). Transversal cross sections were measured at different axial distances where the axial pressure field along the first maximum $x=0.45 \lambda(0.3 \mathrm{~mm})$ was evaluated. The corresponding axial pressure distribution is shown in Fig. 5(c) where the experiment agrees well with the theory. The experimental measurements and numerical integration of both the pressure field and the phase profiles in the transverse planes are shown in Figs. $5(\mathrm{~d})-5(\mathrm{~g})$, $5(\mathrm{i})-5(\mathrm{l})$, and $5(\mathrm{n})-5(\mathrm{q})$ at distances of $z_{1}=8.8 \lambda(6 \mathrm{~mm})$, $z_{2}=17.6 \lambda(12 \mathrm{~mm})$, and $z_{1}=26.5 \lambda(18 \mathrm{~mm})$, respectively. The experimental magnitude of the pressure field matches the characteristics of the first-order Bessel beams with null amplitude on axis and a set of rings of pressure maxima with increasing radii, which shows good agreement with the theory. 

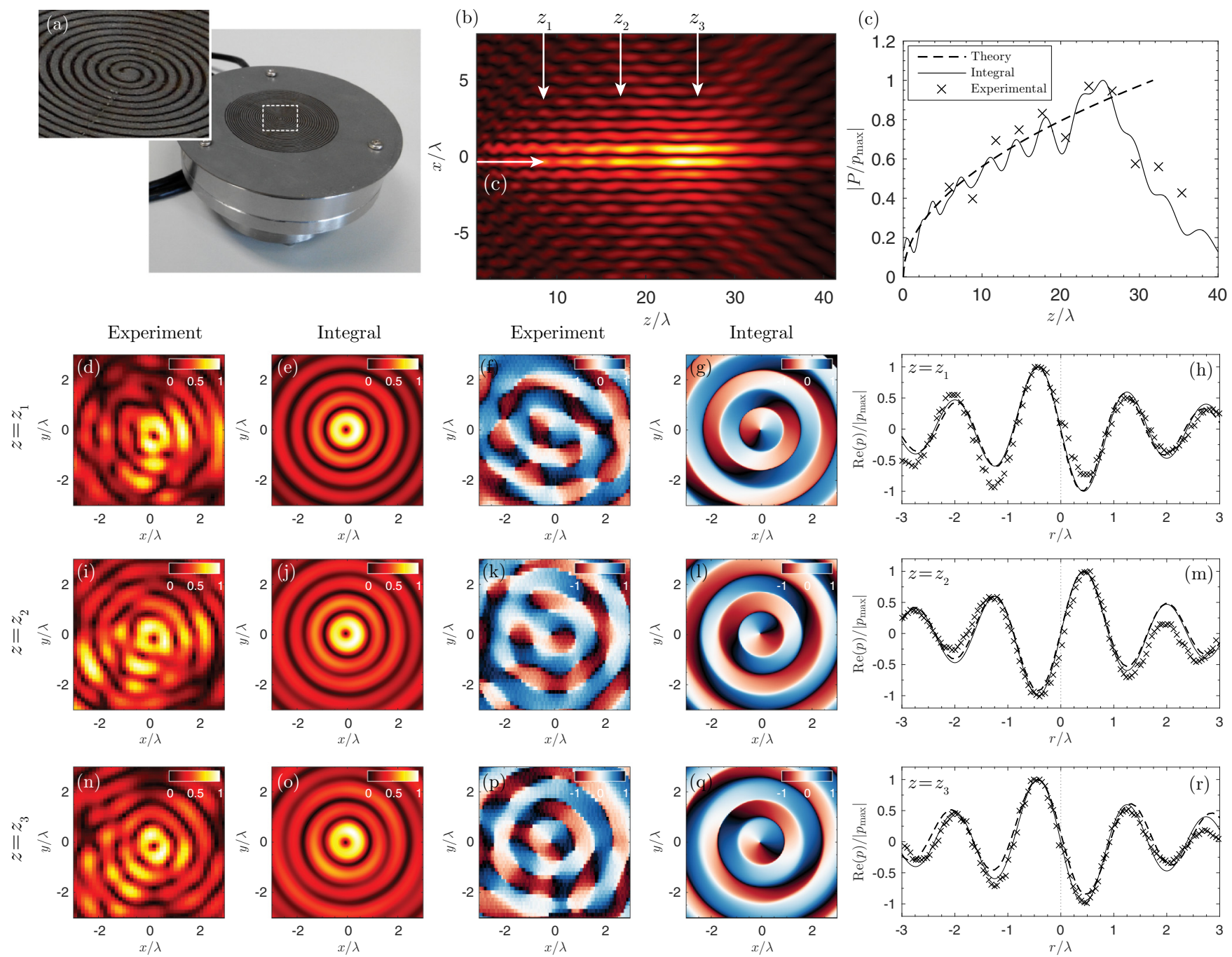

FIG. 5. (a) Spiral grating used in experiments. The inset shows a zoom were the spiral pattern can clearly be seen. (b) Pressure map distribution obtained by numerical integration of the Rayleigh-Sommerfeld integral. (c) Axial pressure distribution at $x=0.45 \lambda$ obtained by the Rayleigh integral for the finite aperture grating (continuous), theoretical for infinite aperture grating (dashed), and experiments (markers). (d), (i), and (n) Experimental field magnitude and (e), (j), and (o) theoretical cross sections at $z_{1}=8.8 \lambda(6 \mathrm{~mm}), z_{2}=17.6 \lambda(12 \mathrm{~mm})$, and $z_{1}=26.5 \lambda(18 \mathrm{~mm})$, respectively; (f), (k), and (p) and (g), (1), and (q) corresponding phases. Color bars in normalized units $\left(p / p_{0}\right.$ for the field magnitude and $\phi / \pi$ for the phase). (h), (m), and (r) Traversal beam cross sections at different axial distances obtained by the Rayleigh integral for the finite aperture grating (continuous), analytic expression of the first-order Bessel beam (dashed), and experiments (markers). The vertical dotted line represents the position where the field is null.

Some differences between theory and experiments appear, that are mainly caused due to technical imperfections: First, the resonances between the grating and the piezoelectric source cause beam distortion and aberrations: there exist multiple reflections in the cavity formed between the spiral plate and the source, and secondary diffracted beams with progressively lower amplitude are generated contributing to vortex beam aberration. There exist other sources of aberration, including the misalignment among the grating, the transducer, and the nonuniform vibration of the piezoelectric element, which is constrained by the manufacturing process of the transducer.

The phase of the field presents the characteristic screw phase dislocation at the center of the beam: A complete loop around a point centered on the axis represents a linear and continuous variation of the phase from 0 to $2 \pi$, i.e., the topological charge of the acoustic vortex is one. We remark that a shift of $\pi$ in phase is observed between any point and its image with respect to the central axis of the plane containing the spiral. This result proves that the wave transmitted through the grating is therefore an acoustic vortex.

Finally, transversal cross sections of the pressure field are shown in Figs. 5(h), 5(m), and 5(r) for the distances of $z_{1}, z_{2}$, and $z_{3}$, respectively. The transversal cross section in the experiments (markers) was chosen for an azimuthal angle on the $(y, x)$ plane at $\theta=70^{\circ}, 30^{\circ}$, and $-51^{\circ}$. The traversal profiles agree well with the shape of the first-order Bessel beam (continuous black line) and the finite aperture prediction by the numerical integration of the Rayleigh-Sommerfeld equation. Although minor differences are visible, the main features of the HOBB, i.e., its central zero and rotational vortices, are correctly reproduced by the proposed experimental setup. 


\section{CONCLUSIONS}

The formation of HOBBs by scattering of plane waves on an Archimedes' spiral grating is theoretically and experimentally reported in this paper. The effect of the finite size of the sample is analyzed numerically. The main result is that, due to finite size of the sample, the HOBBs are truncated. All the beams analyzed are characterized by a zero of the pressure field along the $z$ axis, i.e., the vortex line. The size of this hollow part of the beam is dependent on the topological charge of the HOBB, which is controlled directly by the number of arms of the spiral. Experimental tests in the ultrasound regime have been performed showing the case of a truncated first-order Bessel beam. Good agreement among theory, experimental measurements, and numerical simulations is found for the acoustic pressure field amplitude as well as for the screw phase dislocations. Therefore, the system shown in this paper seems to be of special interest for the generation of arbitrary $n$ th-order Bessel beams using regular spiral patterns with $n$ arms.

The system shown in this paper to synthesize HOBBs presents a high potential in ultrasound particle manipulation techniques and, in general, in acoustic radiation force applications in which the HOBBs have attracted great interest. This method provides the possibility of generation of Bessel beams of arbitrary order by a passive and cheap device if compared with acoustical vortices generated by active arrays of transducers. The generation of an acoustic vortex by active arrays is limited by the amount of active elements and its size. In contrast, the beam resolution by the proposed setting is limited only by the ratio between the wavelength and the spacing between slits. It is worth noting here that a modification of the present device based on coiling slits was presented simultaneously with the present paper [41]. By not using Archimedes spirals the vortex properties can be controlled by passive devices. Nowadays, with the increase in the performance of $3 \mathrm{D}$ printing and laser cutting techniques, the conformation of HOBBs by spiral gratings offers an alternative to multielement transducers to generate acoustical vortices.

\section{ACKNOWLEDGMENTS}

We acknowledge financial support from MINECO of the Spanish Government under Grants No. MTM2012-36740C02-02, No. FIS2015-65998-C2-1-P, and No. FIS201565998-C2-2-P. N.J. acknowledges financial support from PAID-2011 Universitat Politècnica de València.
[1] J. Durnin, J. J. Miceli, Jr., and J. H. Eberly, Phys. Rev. Lett. 58, 1499 (1987).

[2] A. Vasara, J. Turunen, and A. T. Friberg, J. Opt. Soc. Am. A 6, 1748 (1989).

[3] J. Arlt and K. Dholakia, Opt. Commun. 177, 297 (2000).

[4] S. S. R. Oemrawsingh, J. A. W. van Houwelingen, E. R. Eliel, J. P. Woerdman, E. J. K. Verstegen, J. G. Kloosterboer, and G. W. 't Hooft, Appl. Opt. 43, 688 (2004).

[5] P. L. Marston, J. Acoust. Soc. Am. 120, 3518 (2006).

[6] F. Mitri, Ultrasonics 49, 794 (2009).

[7] N. Jiménez, V. Romero-García, R. Picó, A. Cebrecos, V. J. Sánchez-Morcillo, L. Garcia-Raffi, J. V. Sánchez-Pérez, and K. Staliunas, Europhys. Lett. 106, 24005 (2014).

[8] N. Jiménez, V. Romero-García, R. Picó, L. Garcia-Raffi, and K. Staliunas, Appl. Phys. Lett. 107, 204103 (2015).

[9] C. Pfeiffer and A. Grbic, Phys. Rev. B 91, 115408 (2015).

[10] D. Baresch, J.-L. Thomas, and R. Marchiano, Phys. Rev. Lett. 116, 024301 (2016).

[11] N. Heckenberg, R. McDuff, C. Smith, and A. White, Opt. Lett. 17, 221 (1992).

[12] A. Y. Bekshaev and A. Karamoch, Opt. Commun. 281, 3597 (2008).

[13] A. Ashkin, Phys. Rev. Lett. 24, 156 (1970).

[14] A. Ashkin, J. Dziedzic, and T. Yamane, Nature (London) 330, 769 (1987).

[15] A. Ashkin, J. Dziedzic, J. Bjorkholm, and S. Chu, Opt. Lett. 11, 288 (1986).

[16] D. G. Grier, Nature (London) 424, 810 (2003).

[17] L. Zhang and P. L. Marston, Phys. Rev. E 84, 065601 (2011).

[18] Z. Y. Hong, J. Zhang, and B. W. Drinkwater, Phys. Rev. Lett. 114, 214301 (2015).
[19] P. L. Marston, J. Acoust. Soc. Am. 122, 3162 (2007).

[20] J. Nye and M. Berry, Proc. R. Soc. London, Ser. A 336, 165 (1974).

[21] B. T. Hefner and P. L. Marston, J. Acoust. Soc. Am. 106, 3313 (1999).

[22] J. L. Ealo, J. C. Prieto, and F. Seco, IEEE Trans. Ultrason. Ferroelectr. Freq. Control 58, 1651 (2011).

[23] S. Gspan, A. Meyer, S. Bernet, and M. Ritsch-Marte, J. Acoust. Soc. Am. 115, 1142 (2004).

[24] X. Jiang, Y. Li, B. Liang, J.-c. Cheng, and L. Zhang, Phys. Rev. Lett. 117, 034301 (2016).

[25] J.-L. Thomas and R. Marchiano, Phys. Rev. Lett. 91, 244302 (2003).

[26] C. Demore, Z. Yang, A. Volovick, H. Wang, S. Cochran, M. MacDonald, and G. Spalding, in 2011 IEEE International Ultrasonics Symposium (IUS) (IEEE, Piscataway, NJ, 2011), pp. 180-183.

[27] J. Wu, J. Acoust. Soc. Am. 89, 2140 (1991).

[28] T. Wang, M. Ke, S. Xu, J. Feng, C. Qiu, and Z. Liu, Appl. Phys. Lett. 106, 163504 (2015).

[29] A. Marzo, S. A. Seah, B. W. Drinkwater, D. R. Sahoo, B. Long, and S. Subramanian, Nat. Commun. 6, 8661 (2015).

[30] K. Volke-Sepúlveda, A. O. Santillán, and R. R. Boullosa, Phys. Rev. Lett. 100, 024302 (2008).

[31] K. Skeldon, C. Wilson, M. Edgar, and M. Padgett, New J. Phys. 10, 013018 (2008).

[32] C. Yoon, B. J. Kang, C. Lee, H. H. Kim, and K. K. Shung, Appl. Phys. Lett. 105, 214103 (2014).

[33] P. Li, Z. Mao, Z. Peng, L. Zhou, Y. Chen, P.-H. Huang, C. I. Truica, J. J. Drabick, W. S. El-Deiry, M. Dao et al., Proc. Natl. Acad. Sci. USA 112, 4970 (2015). 
[34] F. Guo, Z. Mao, Y. Chen, Z. Xie, J. P. Lata, P. Li, L. Ren, J. Liu, J. Yang, M. Dao et al., Proc. Natl. Acad. Sci. USA 113, 1522 (2016).

[35] B. Raiton, J. McLaughlan, S. Harput, P. Smith, D. Cowell, and S. Freear, Appl. Phys. Lett. 101, 044102 (2012).

[36] T. Brunet, J.-L. Thomas, R. Marchiano, and F. Coulouvrat, New J. Phys. 11, 013002 (2009).

[37] F. G. Mitri, IEEE Trans. Ultrason. Ferroelectr. Freq. Control 61, 2089 (2014).
[38] C. Paterson, Opt. Commun. 124, 121 (1996).

[39] K. Firouzi, B. Cox, B. Treeby, and N. Saffari, J. Acoust. Soc. Am. 132, 1271 (2012).

[40] M. Abramowich and I. A. Stegun, Handbook of Mathematical Functions with Formulas, Graphs and Mathematical Tables (Dover, New York, 1972).

[41] X. Jiang, J. Zhao, S.-1. Liu, B. Liang, X.-y. Zou, J. Yang, C.-W. Qiu, and J.-c. Cheng, Appl. Phys. Lett. 108, 203501 (2016). 